\title{
Mid-infrared spectrum of the zodiacal light observed with ISOPHOT *
}

\author{
Ch. Leinert ${ }^{1}$, P. Ábrahám ${ }^{1,2}$, J. Acosta-Pulido ${ }^{3}$, D. Lemke ${ }^{1}$, and R. Siebenmorgen ${ }^{4}$ \\ 1 Max-Planck-Institut für Astronomie, Königstuhl 17, 69117 Heidelberg, Germany \\ 2 Konkoly Observatory of the Hungarian Academy of Sciences, PO Box 67, 1525 Budapest, Hungary \\ 3 Instituto de Astrofísica de Canarias, via Láctea s/n, 38200 La Laguna, Tenerife, Spain \\ ${ }^{4}$ European Southern Observatory, Karl-Schwarzschild-Strasse 2, 85748 Garching b. München, Germany
}

Received 2 September 2001 / Accepted 11 July 2002

\begin{abstract}
We present 29 mid-infrared spectra of the zodiacal light distributed over the sky. The observed 5.9-11.7 $\mu \mathrm{m}$ spectral shapes are well represented by blackbody radiation with colour temperatures in the range of $255 \leq T \leq 300 \mathrm{~K}$. The spectra are smooth and featureless. The variation of the temperature can be explained by the geometrical distribution of dust in the inner solar system. This result indicates that although the interplanetary dust particles originate from discrete sources (comets, asteroids) the interplanetary cloud of today seems to be well mixed in terms of grain composition and size distribution.
\end{abstract}

Key words. interplanetary medium - infrared: solar system

\section{Introduction}

The spectral energy distribution of the zodiacal light is dominated by scattered sunlight at $\lambda \leq 3.5 \mu \mathrm{m}$ and by thermal emission of dust grains at longer wavelengths. The infrared part of the spectrum, which cannot be observed from the ground, was measured in broad photometric bands from rockets and balloons (Soifer et al. 1971; Price et al. 1980; Murdock \& Price 1985; Salama et al. 1987), and from the IRAS and COBE satellites (Hauser et al. 1984; Reach et al. 1996a). These data sets were successfully used to deduce the spatial structure of the interplanetary dust cloud.

One of the most interesting parts of the zodiacal light spectrum is the mid-infrared $(5-16 \mu \mathrm{m})$ wavelength range, where the surface brightness increases by two orders of magnitudes. In addition to the practical importance of characterizing this steep rise, this is the spectral regime to look for spectral signatures produced by the smallest particles in the interplanetary dust cloud and to identify their basic constituents (Röser \& Staude 1978; Reach 1988). Before the launch of the Infrared Space Observatory (ISO, Kessler et al. 1996) only one rocket measurement of the mid-infrared spectrum was attempted (Briotta 1976). From ISO Reach et al. (1996b)

Send offprint requests to: $\mathrm{Ch}$. Leinert,

e-mail: leinert@mpia-hd.mpg.de

* Based on observations with ISO, an ESA project with instruments funded by ESA member states (especially the PI countries France, Germany, The Netherlands and the UK) with participation of ISAS and NASA. observed the spectrum at one sky position with the ISOCAM circular variable filters $(\lambda / \Delta \lambda \approx 40)$ from 5 to $16 \mu \mathrm{m}$. They found that the spectrum is remarkably well fitted by a Planck curve of $260 \mathrm{~K}$. There were no indications for sharp lines, but a broad hump in the 9-11 $\mu \mathrm{m}$ range was detected at the $10 \%$ level, tentatively identified with emission of silicate particles. They also reported a good agreement with the COBE/DIRBE broad-band photometry at $4.9 \mu \mathrm{m}$ and $12 \mu \mathrm{m}$. Recently a new measurement of the near- and mid-infrared spectrum of the zodiacal light was performed by the IRTS satellite (Ootsubo et al. 2000). They claim that the entire spectrum of 3-12 $\mu \mathrm{m}$ cannot be fitted by one gray body emissivity model, and suggest that either the dust emissivity is not constant in this spectral range or there exist a significant population of submicron size very hot particles in the solar system.

Here we present the mid-infrared spectrum of the zodiacal light as observed between $5.9 \mu \mathrm{m}$ and $11.7 \mu \mathrm{m}$ with ISOPHOT$\mathrm{S}(\lambda / \Delta \lambda \approx 95)$, the spectrophotometric subinstrument of ISO's photometer ISOPHOT (Lemke et al. 1996). The first results, derived from a small set of dedicated zodiacal light observations revealed a blackbody-like spectrum with temperature of 264 K (Ábrahám et al. 1997). Since this ISOPHOT-S spectrum is consistent with the ISOCAM and the IRTS results at the $20 \%$ level, the general shape of the mid-infrared spectrum is relatively well determined by now. Very little is known, however, on the variation of the spectral shape over the sky. Such variations may arise from temperature differences within the interplanetary dust cloud, or from differences in dust composition and size distribution in different viewing directions. In this 


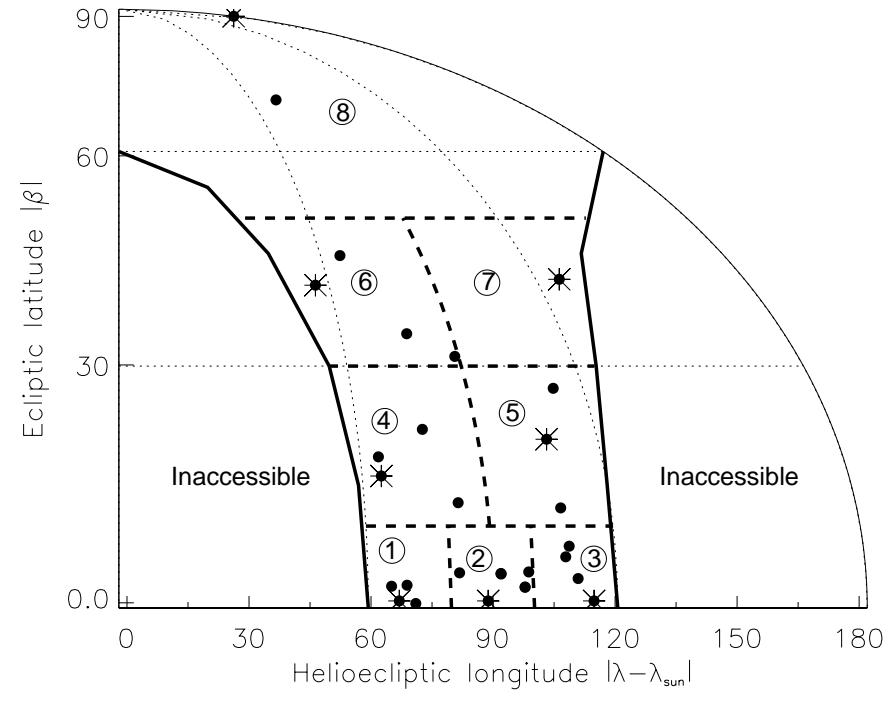

Fig. 1. Distribution on the sky of the positions where ISOPHOT-S spectra were taken. The accessible region of the sky $\left(90^{\circ} \pm 30^{\circ}\right.$ from the sun) has been divided into eight segments of differing latitude and longitude. In each segment a reference spectrum (asterisk) was selected, to which the individual spectra were scaled in absolute brightness before creating "template" spectra by averaging.

paper we analyse a database of 29 ISOPHOT-S spectra distributed over the accessible sky in order to search for possible variations in the spectral shape.

\section{Observations and data reduction}

\subsection{ISOPHOT-S low resolution spectra}

We searched the ISO Archive for long $\left(1024 \mathrm{~s} \leq t_{\text {meas }} \leq\right.$ 4096 s) ISOPHOT-S observations of the sky background, and retrieved 62 spectra from different scientific and calibration programmes. Several measurements were dedicated observations of the zodiacal light (Ábrahám et al. 1997), but most of them were taken as reference measurement close to faint targets. All spectra were obtained in staring mode.

Some of the spectra were discarded from the list either because they were observed at the very beginning of the orbit when the accuracy of dark current subtraction is reduced, or because they suffered from memory effects due to preceding observations of very bright sources. The distribution of the remaining 29 observations on the sky is shown in Fig. 1. For the present study we assume cylindrical symmetry of the zodiacal cloud and neglect the inclination of its symmetry plane to the Ecliptic, thus spectra taken at negative solar helioecliptic longitudes or at negative ecliptic latitudes are also plotted in the first quarter of the sky in Fig. 1.

The basic data processing was performed using the PHT Interactive Analysis PIA_V9.0 (Gabriel et al. 1997). After discarding cosmic ray hits, linear fits were performed to the integration ramps, and a dark current signal depending on the orbital phase was subtracted. The next standard calibration step for ISOPHOT-S is a transformation between the measured signal and the flux density: Flux $=\tau($ Signal, $t)$ where Flux is in (Jy), Signal is in $(\mathrm{V} / \mathrm{s})$, and $t$ is the time measured from the start of the observation. The $\tau($ Signal, $t)$ relationship was determined for each of the 128 detector pixels independently, mainly based on a set of standard star measurements, and the results are stored in one of the ISOPHOT calibration (CalG) files.

The standard relationships, however, cannot be used to calibrate our zodiacal light measurements. At the lowest signal level these relationships were constrained by sky background measurements rather than by standard star observations. Since the flux prediction for these background measurements is a blackbody model fitted to the DIRBE 4.9 and $12 \mu \mathrm{m}$ photometry, applying the standard calibration to our observations would give back this model within the measurement uncertainties. Thus for the purpose of the present study we had to modify the content of the CalG file, basing it exclusively on standard star observations with ISOPHOT.

In general, the function $\tau($ Signal, $t)$ is a non-linear function of the signal, particularly at high signal level while faint signals may be expected to be linear. Anyway, since there are only three faint standard stars observed with long measurement time $\left(t_{\text {meas }}=1024 \mathrm{~s}\right)$, constraining a non-linear function by these three observations would not be possible. Instead we make the assumption that the function $\tau($ Signal, $t)$ is linear in the signal range of the zodiacal light observations, i.e. for a given integration time $t$ we have Flux $=R \times$ Signal and the only parameter to be determined pixel by pixel is the proportionality factor $R$. In order to demonstrate that this linearity assumption is realistic, we plotted in Fig. 2a the ISOPHOT-S signals for pixel No. $100(9.2 \mu \mathrm{m})$ of the 29 sky background measurements vs. the DIRBE broad-band photometry at $12 \mu \mathrm{m}$ for the same sky positions. The clear linear relationship between the two instruments with practically no difference in the zero points indicates the usefulness of our calibration procedure, and shows that as far as the DIRBE calibration is linear the ISOPHOT-S signals are linear, too.

As a first approximation in deriving the constant $R=$ Flux/Signal we took a value from the standard CalG file corresponding to a higher signal level, determined by standard stars rather than sky background measurements, and used this ratio at the signal level of the zodiacal light observations, too. We chose a signal level of 5 times the signal of the brightest zodiacal light measurement, because at this signal level $R$ is practically independent of the background flux prediction adopted (it changes by less than $10 \%$ for a $50 \%$ variation of the background flux).

In order to test the applicability of this linearized version of the ISOPHOT-S calibration, we evaluated the On and Off observations of the 3 faint standard star of $t_{\text {meas }}=1024 \mathrm{~s}$, and compared the results with model predictions for their photospheric radiation. Figure $2 b$ shows that there is no wavelength dependent effect, but the ISOPHOT-S flux densities are systematically below the expectations by about $10-15 \%$. Since this underestimation factor seems to be relatively constant over all wavelengths, we decided to apply a constant correction factor of $1 / 0.88$ for all pixels and for all zodiacal light measurements.

To convert this calibration from point sources to ectended brightness, the effective solid angle per pixel must be known. With an earlier version of the data processing, including the then standard values of effective solid angle, we had obtained a 

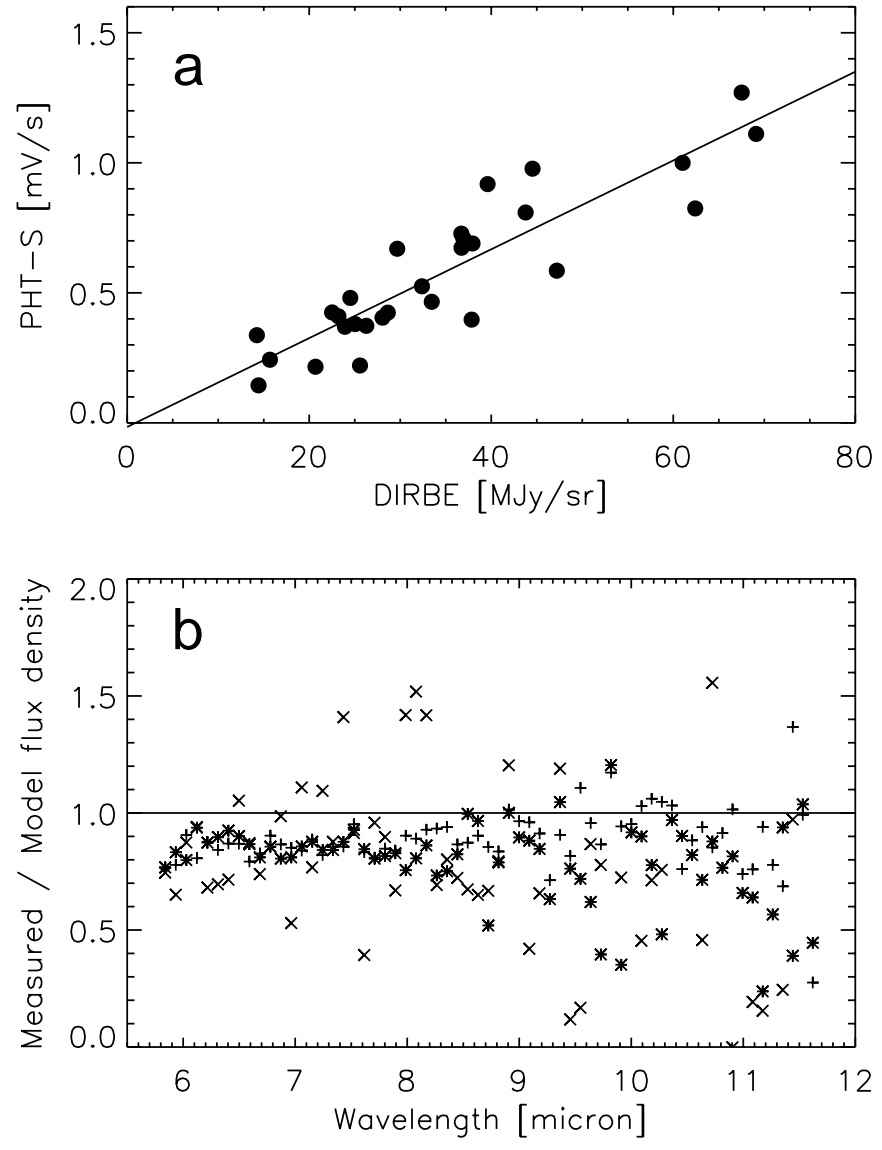

Fig. 2. a) Existence of a linear relationship between the ISOPHOT$\mathrm{S}$ raw signals, measured with Pixel $100(9.2 \mu \mathrm{m})$ at $t=1204 \mathrm{~s}$, and surface brightness values at $12 \mu \mathrm{m}$ extracted from the DIRBE Weekly Sky Maps. b) Comparison of the ISOPHOT-S spectra of the 3 faint standard stars, calibrated with a modified linear version of the OLP10 CalG file, with atmospheric model predictions. Plus sign: HD $211476(K=5.55 \mathrm{mag})$, asterisk: HR 8487 ( $K=5.51 \mathrm{mag})$, cross: HD 184400 ( $K=7.54 \mathrm{mag})$.

weak emission feature in the spectra around $9.3 \mu \mathrm{m}$ (Ábrahám et al. 1999). The presence of this feature is not affected by the modifications in faint point source calibration discussed above. However, since its the shape resembled an instrumental characteristics curve, namely the variation of the effective solid angle with wavelength (dashed line in Fig. 3), we re-analysed all ISOPHOT-S beam profile measurements in order to check for a possible instrumental origin. In addition to the dedicated scan observations on HR 6705 (Rev.342), which had exclusively been used previously to create the beam profile for the official ISOPHOT Off-Line Processing (OLP) software (see ISOPHOT Handbook, Laureijs et al. 2001), we considered also the available maps centered on HR 7924 (Revs. 53 and 154) as well as 1-dimensional scans crossing $3 \mathrm{CVn}$ and CF Boo (Rev. 20). We were able to derive a beam profile per pixel which was consistent with the additional measurements, but the results disagreed with the ones adopted in the OLP (for the outer part of the map where no measurements were available the theoretical beam profile was adopted). The differences between the new and the OLP effective solid angle values are displayed in Fig. 3. We have no convincing explanation of the mismatch between

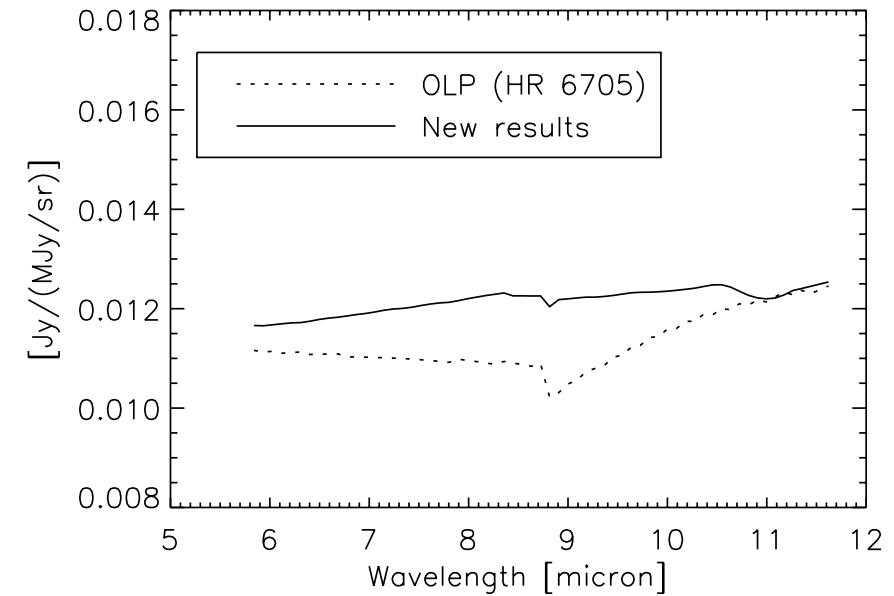

Fig. 3. Comparison of the effective solid angle values of ISOPHOT-S adopted in the Off-Line Processing software (dashed line) with the new values derived in our analysis in Sect. 2.1 (solid line).

the two data sets. However, since the results derived from the additional measurements are based on a larger set of observations, including also two-dimensional maps, in the present paper we adopt the new results.

\subsection{ISOCAM parallel mode data}

Whenever an ISOPHOT-S observation was performed the infrared camera ISOCAM (Cesarsky et al. 1996) also exposed to the sky and collected data in a direction separated by $12^{\prime}$ from the ISOPHOT position. Since such a positional offset is of no importance for our zodiacal light studies, the ISOCAM parallel mode provided us with one or two filter band measurements for each spectrum, centred at $6.0 \mu \mathrm{m}$ (LW4) and/or $6.7 \mu \mathrm{m}$ (LW2). We used these measurements for an independent cross-check of the final calibration accuracy of the ISOPHOT-S spectra. The data processing steps as well as a more detailed description of the ISOCAM parallel mode are presented in Siebenmorgen et al. $(1996,2000)$.

\section{Results}

In order to search for variations of the spectral shape over the sky, we divided the sky region accessible to ISO into eight segments as indicated in Fig. 1. The segments contain 1-5 target positions. In order to increase the signal-to-noise ratio, we averaged the spectra in each sky segment and derived a "template" for that part of the sky. In each segment a reference spectrum (marked by asterisk in Fig. 1) was selected and before the averaging procedure all spectra in the segment were scaled to the absolute brightness level of the reference one. Figure 4 displays the eight template spectra; the error bars on the individual pixels correspond to $1 \sigma$ standard deviation of the averaged spectra of the segment after scaling. In the figure we also overplotted the ISOCAM Parallel Mode data as well as the COBE/DIRBE 4.8 and $12 \mu \mathrm{m}$ photometric points extracted from the Weekly Sky Maps. The comparison of the ISOPHOT-S, ISOCAM and DIRBE data confirms that the absolute photometric calibrations of the three instruments are consistent. 


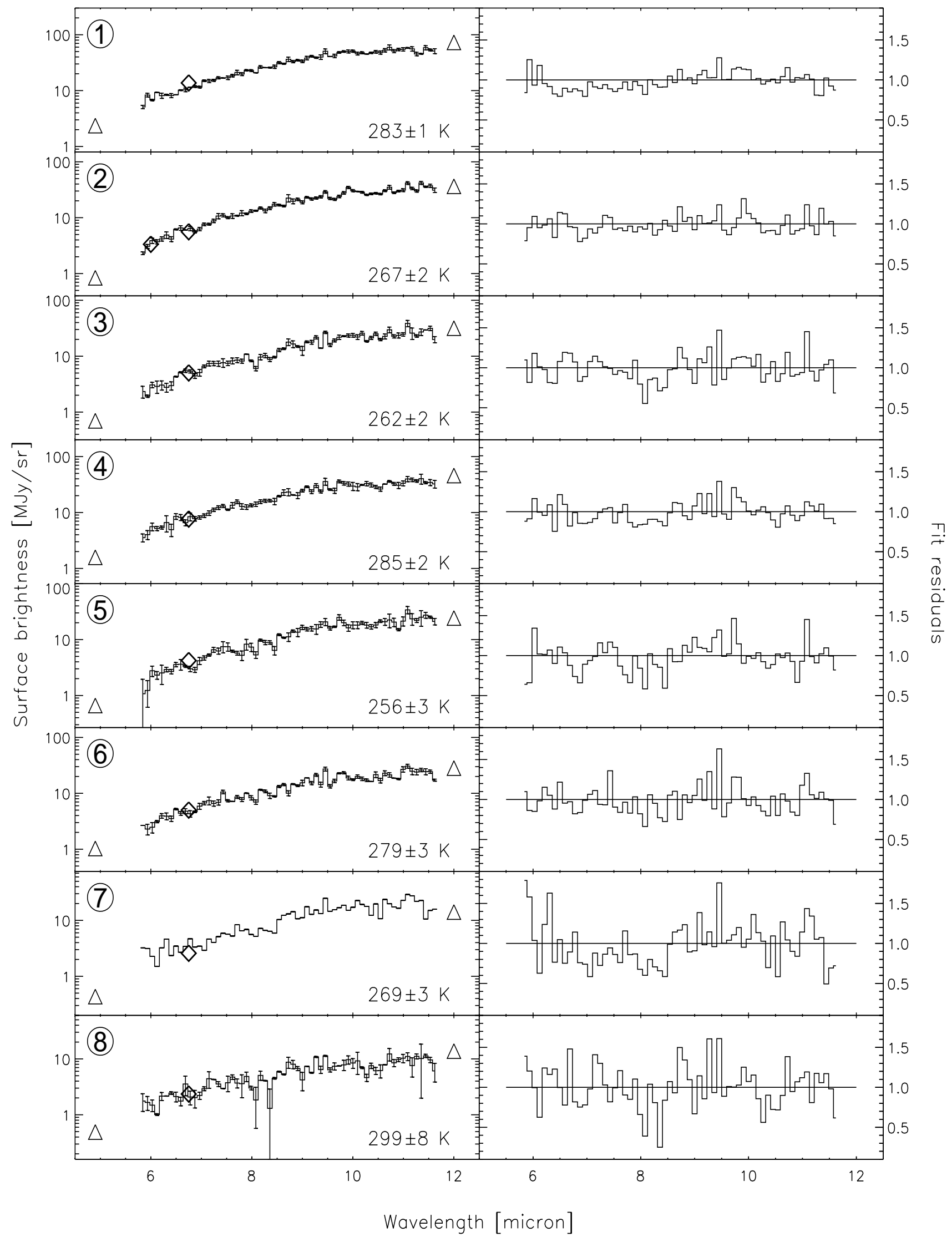

Fig. 4. ISOPHOT-S spectra of the zodiacal light (solid lines). Left panel: template spectra derived by averaging the individual spectra in each of the sky segments defined in Fig. 1. The temperature values are derived from blackbody fits to the templates; the errors correspond to the formal uncertainties given by the fitting procedure. Triangles: DIRBE 4.9 and $12 \mu \mathrm{m}$ photometry, Diamonds: ISOCAM parallel mode data. Right panel: residuals with respect to the blackbody fits. 
All templates can be well approximated by single temperature Planck curves, whose temperatures as well as the fit residuals - given as the ratio between observation and fit - are given in Fig. 4. Equal weighting of data points was applied in the fit; the uncertainties correspond to the formal error bars determined by the fitting routine. The derived temperatures show systematic variations along the sky: the temperature value increases with the ecliptic latitude and decreases with the distance from the sun. The highest temperatures are observed towards the ecliptic poles, as expected for a flattened dust distribution, where the average heliocentric distance of the dust particles is smaller in this viewing direction than for similar line-of-sights at low ecliptic latitudes.

In Fig. 5 we compare the temperature values derived from the blackbody fits with the colour temperatures computed from the DIRBE $4.9 / 12 \mu \mathrm{m}$, and $12 / 25 \mu \mathrm{m}$ photometric data for the same sky positions after colour correction. The DIRBE 4.9/12 colour temperatures are higher (on the average by $6 \mathrm{~K}$ ), while the $12 / 25$ colour temperatures are lower (on the average by $7 \mathrm{~K}$ ) than the corresponding ISOPHOT-S values. A possible explanation for the first result is that the thermal emission of interplanetary dust in the DIRBE $4.9 \mu \mathrm{m}$ measurement is contaminated to a larger extent with scattered sunlight or perhaps also galactic background emission than the ISOPHOT-S spectra, which start at a somewhat longer wavelength of $5.9 \mu \mathrm{m}$. A difference of $10 \%-15 \%$ could be expected from the first effect, just sufficient to explain the higher $4.9 / 12 \mu \mathrm{m}$ colour temperatures. The lower DIRBE 12/25 $\mu \mathrm{m}$ colour temperatures could be an indication that longwards of $12 \mu \mathrm{m}$ the zodiacal spectrum slightly deviates from the blackbody curve. However, we should be cautious with such a conclusion: this spectral regime was not sampled with ISOPHOT-S, and temperature is less accurately defined over this range, e.g. only $5 \%$ change in the $12 / 25 \mu \mathrm{m}$ brightness ratio already would account for the temperature difference we are discussing here.

In Fig. 6 we averaged the fit residuals for all templates, in order to increase the signal-to-noise ratio and to search for possible weak spectral features. The standard deviation of the points around ratio $=1$ is $\sigma=0.12$. There is no evidence for a systematic deviation from the fits. We conclude that within the measurement accuracy we achieved (12\%) no significant spectral feature can be observed in the average spectrum of the zodiacal light.

\section{Discussion}

Our motivation to study the variation of the mid-infrared spectrum of the zodiacal light over the sky was to learn if interplanetary dust particles of different origin were distinguishable by their observed properties and would show up in our zodiacal light spectra. It could be expected that the spectrum of dust at high ecliptic latitudes should show similarities to the spectrum of comets of high inclination, while properties of dust close to the Ecliptic Plane could resemble those of the asteroids. Also, interstellar dust particles entering the solar system, as identified by the dust experiment on the Ulysses space probe (Grün et al. 1993) could leave their imprint in the data. Indeed, Renard et al. (1995), based on IRAS observations of the
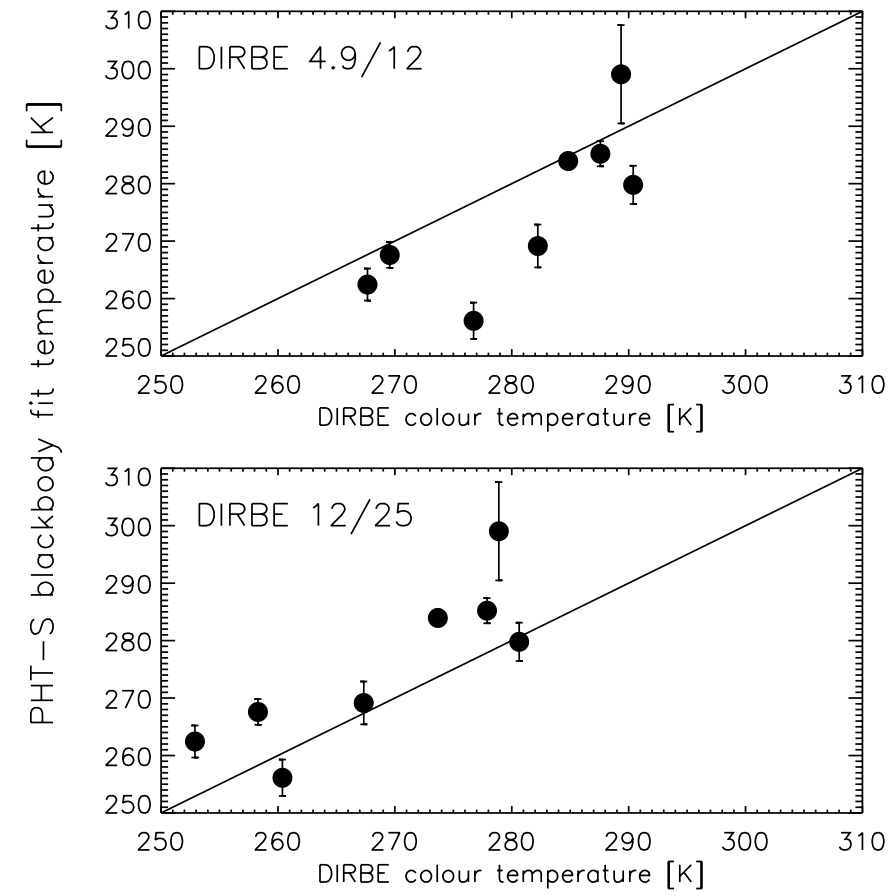

Fig. 5. Comparison of the colour temperatures derived from blackbody fits to the ISOPHOT-S spectra with the DIRBE $[4.9,12] \mu \mathrm{m}$ and $[12,25] \mu \mathrm{m}$ colour temperatures. The solid line marks equality between these two temperatures.

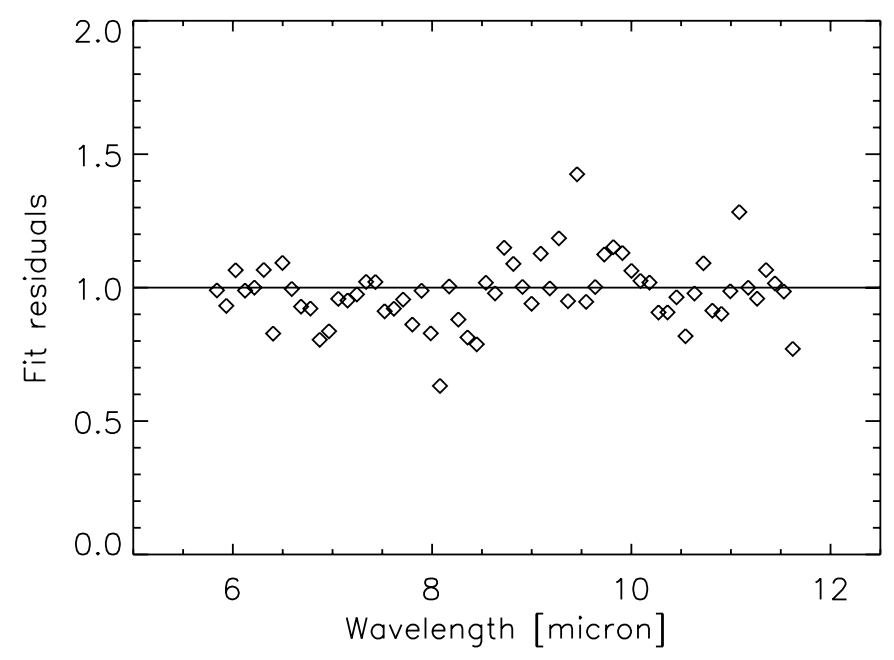

Fig. 6. Fit residuals averaged for all template spectra.

zodiacal light, concluded that there should be at least two different populations: a low albedo, high polarisation one close to the ecliptic, and a more randomly distributed component with higher albedo and lower polarisation (Levasseur-Regourd 1991), and they suggested to identify these with particles of asteroidal and cometary origin, respectively. As already mentioned, Reach et al. (1996b) pointed out the possibility that a silicate feature around $10 \mu \mathrm{m}$ might be present in the zodiacal light spectrum. Regions with stronger feature would then be identified as regions containing relatively larger numbers of small warm silicate particles. 

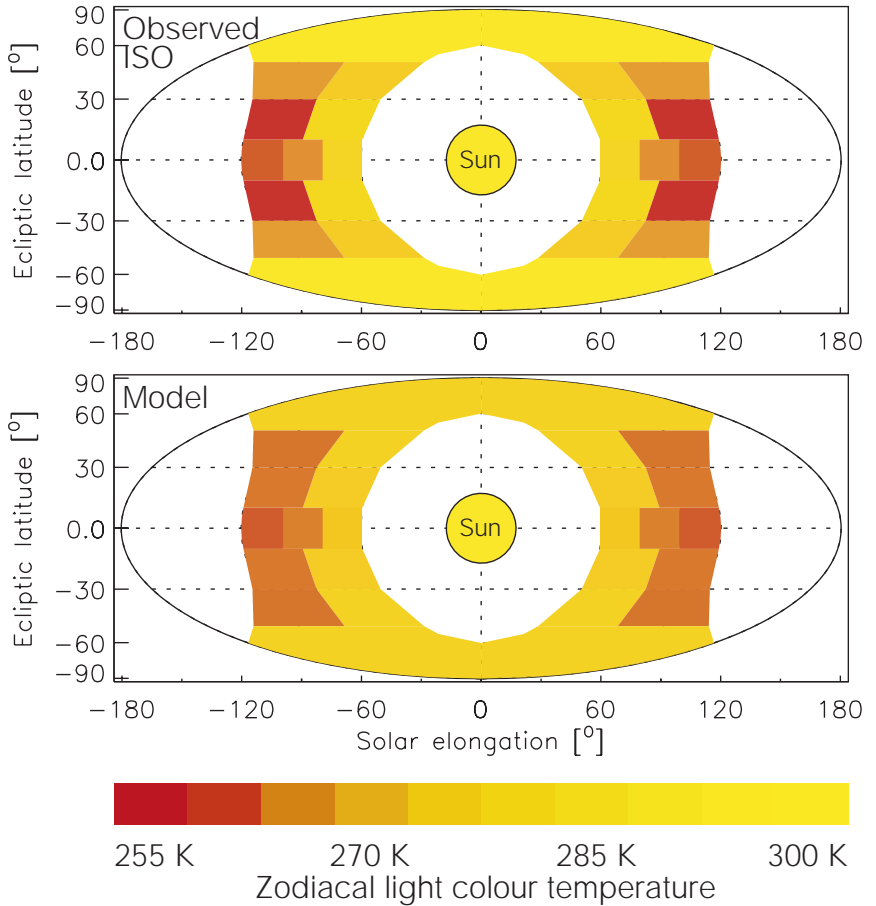

Fig. 7. Upper panel: colour temperatures derived from the ISOPHOT-S templates using single temperature blackbody fits. Lower panel: colour temperatures predicted by our model in the spectral range of ISOPHOT-S.

Our ISOPHOT-S data taken at 29 different positions over the sky, however, do not show any significant deviation from blackbody radiation, and no significant variation in the spectra other than temperature effects. The residuals of the blackbody fits, plotted on the right hand side of Fig. 4 and averaged in Fig. 6, do not show any definite spectral features as they might arise from PAHs, ices or crystalline or amorphous silicates. In particular, the lack of a strong $10 \mu \mathrm{m}$ feature in the observed spectrum, which is well observed in comets (e.g. Hanner et al. 1994; Crovisier 2000), rules out the presence of a significant population of very small amorphous silicate grains in interplanetary space. Because of this lack of obvious spectral features, it appears that the origin of the particles cannot be determined from their mid-infrared spectrum at the level of the present $10 \%$ to $20 \%$ accuracy. Conversely, this result suggests that the interplanetary dust cloud at present is well mixed.

Thus, we expect that the measured temperature distribution may simply reflect the geometrical distribution of dust within the interplanetary dust cloud. In order to investigate this question we constructed a simple but representative geometrical model of the interplanetary dust cloud, which we then used to predict the temperature distribution naturally resulting from purely geometrical effects.

For the modelling, we assumed the interplanetary dust particles to emit blackbody radiation at the local equilibrium temperature (at $1 \mathrm{AU} T=280 \mathrm{~K}$ was assumed). In view of the low albedo, $\approx 0.08$ at the earth's orbit (Hauser \& Houck 1986; Renard et al. 1995), and in view of the large effective size of the particles of $\approx 60 \mu \mathrm{m}$ diameter (Grün et al. 1985; Leinert \& Grün 1990), this will be quite a reasonable approximation. Scattering can be neglected in our wavelength range of $\lambda \geq 6 \mu \mathrm{m}$. For the spatial distribution of interplanetary dust we take a separated two-parameter representation $n(r, \beta)=n(r) f(\beta)$, where $r$ is the heliocentric distance of the particles and $\beta$ the heliocentric ecliptic latitude. This certainly is adequate to describe the large-scale properties of zodiacal light in which we are interested here. Absolute normalisation is not necessary since we only need spectral shapes to derive temperatures. For radial dependences, we use $n(r)=r^{-0.93}$ (Renard et al. 1995), and from the same authors the dust particle temperature distribution $T \sim r^{-0.36}$. Such a gradient can result if the emissivity of the particles systematically decreases with wavelength. For the out-of-ecliptic decrease as function of heliocentric ecliptic latitude, the form $f\left(\beta_{\odot}\right) \sim \mathrm{e}^{\alpha\left|\sin \left(\beta_{\odot}\right)\right|^{1.3}}$ (Leinert et al. 1976) was used, which had been successful in reproducing the eclipticto-polar-meridian zodiacal light brightness profiles, and which fits well the COBE and ISOPHOT measurements along the $90^{\circ}$ ecliptic-to-pole arc with a value of $\alpha=-4.8$.

Using this standardised model, we calculated, along representative line-of-sights for the different sky segments shown in Fig. 1, the relative brightnesses of zodiacal light between $5 \mu \mathrm{m}$ and $12 \mu \mathrm{m}$ with a spectral resolution similar to that of the observations. These synthetic spectra were fitted with Planck functions in the same way as we did for the observations. The resulting predicted large scale temperature distibution is very similar to the observed one, showing that in a first approximation the measured spectral variations can be understood in terms of purely geometrical effects of the dust distribution within the interplanetary dust cloud (Fig. 7), without the need to invoke substantial inhomogeneities in particle properties.

\section{Summary}

From an analysis of 29 ISOPHOT-S mid-infrared spectra, distributed over the sky, we found:

- The 5.9-11.7 $\mu \mathrm{m}$ spectral shapes can be well represented by blackbody radiation.

- The colour temperatures derived from these single temperature fits to the spectra are close to the COBE/DIRBE $4.9 / 12 \mu \mathrm{m}$ and $12 / 25 \mu \mathrm{m}$ colour temperatures.

- The temperature variations over the sky, observed in the $255-300 \mathrm{~K}$ range, can be understood in terms of geometrical effects.

- The obvious lack of variations in the mid-infrared spectral shape of the zodiacal light other than due to these temperature changes gives a strong indication that the interplanetary dust cloud is well mixed in terms of grain composition and size distribution.

Acknowledgements. This work was supported by the Max Planck Society, funds from the DLR (formely DARA), Bonn, and by the grant OTKA T 037508 of the Hungarian Scientific Research Fund. The ISOPHOT Data Centre at MPIA is supported by Deutsches Zentrum für Luft- and Raumfahrt e.V. (DLR) with funds of Bundesministerium für Bildung und Forschung, grant No. 50 QI 02013. 


\section{References}

Ábrahám, P., Acosta-Pulido, J. A., Laureijs, R. J., et al. 1997, in Proc. First ISO Workshop on Analytical Spectroscopy, ESA SP-419, ed. A. M. Heras, K. Leech, N. R. Trams, \& M. Perry, 119

Ábrahám, P., Leinert, Ch., Acosta-Puliso, J. A., Schmidtobreick, L., \& Lemke, D. 1999, Zodiacal light observations with ISO, in The Universe as Seen by ISO, ed. P. Cox, \& M. F. Kessler, ESA SP427,145

Briotta, D. A. Jr. 1976, Ph.D. Thesis, Cornell Univ.

Cesarsky, C. J., Abergel, A., Agnese, P., et al. 1996, A\&A, 315, L32

Crovisier, J. 2000, in Proc. of IAU Symp. 197, ed. Y. C. Minh, \& E. F. van Dishoeck, 461

Gabriel, C., Acosta-Pulido, J., Heinrichsen, I., Morris, H., \& Tai, W.-M. 1997, in Proc. of the ADASS VI Conf., ed. G. Hunt, \& H. E. Payne, 108

Grün, E., Zook, H. A., Fechtig, H., \& Giese, R. H. 1985, Icarus, 62, 244

Grün, E., Zook, H. A., Baguhl, M., et al. 1993, Nature, 362, 428

Hanner, M. S., Lynch, D. K., \& Russell, R. W. 1994, ApJ, 425, 274

Hauser, M. G., \& Houck, J. R. 1986, in Light on Dark Matter, ed. F. P. Israel (Reidel), 39

Hauser, M. G., Gillett, F. C., Low, F. J., et al. 1984, ApJ, 278, 15

Kessler, M. F., Steinz, J. A., Anderegg, M. E., et al. 1996, A\&A, 315, L27

Leinert, Ch., Link, H., \& Pitz, E. 1976, A\&A, 47, 221
Leinert, Ch., \& Grün, E. 1990, in Physics of the Inner Heliosphere, ed. R. Schwenn, \& E. Marsch (Springer-Verlag), 207

Lemke, D., Klaas, U., Abolins, J., et al. 1996, A\&A, 315, L64

Levasseur-Regourd, A.-C., in Origin and Evolution of Interplanetary Dust, ed. A.-C. Levasseur-Regourd, \& H. Hasegawa (Kluwer, 1991), 131

Murdock, T. L., \& Price, S. D. 1985, AJ, 90, 375

Ootsubo, T., Onaka, T., Yamamura, I., et al. 2000, Adv. Space Res., 25,2163

Price, S. D., Murdock, T. L., \& Marcotte, L. P. 1980, AJ, 85, 765

Renard, J. B., Levasseur-Regourd, A.-C., \& Dumont, R. 1995, A\&A, 304,602

Reach, W. T. 1988, ApJ, 335, 468

Reach, W. T., Franz, B. A., Kelsall, T., \& Weiland, J. L. 1996a, in Unveiling the cosmic infrared background, ed. E. Dwek, AIP Conf. Proc., 348, 37

Reach, W. T., Abergel, A., Boulanger, F., et al. 1996b, A\&A, 315, L381

Röser, S., \& Staude, H. J. 1978, A\&A, 67, 381

Salama, A., Andreari, P., dall'Oglio, G., et al. 1987, AJ, 93, 467

Siebenmorgen, R., Abergel, A., Altieri, B., et al. 1996, A\&A, 315, 169

Siebenmorgen, R., Schartel, N., \& Ott, S. 2000, in Proc. of ISO Surveys of a Dusty Universe, ed. D. Lemke, M. Stickel, \& K. Wilke (Springer), 275

Soifer, T. B., Houck, J. R., \& Harwit, M. 1971, ApJ, 168, 73 